\title{
Cardiac remodeling after reduction of high-flow arteriovenous fistulas in end-stage renal disease: methodological issues
}

\author{
Hypertension Research (2017) 40, 412; doi:10.1038/hr.2016.171; published online 1 December 2016
}

We read with great interest the recent article titled 'Cardiac remodeling after reduction of high-flow arteriovenous fistulas in end-stage renal disease' by Wohlfahrt et al. ${ }^{1}$ In this paper, the authors assessed whether an elevated cardiac index (CI) or increased arteriovenous fistula (AVF) flow can more accurately identify subjects with reverse remodeling AVF reduction. They found that the effect of AVF reduction on heart remodeling can be predicted by $\mathrm{CI}$ before surgery but not by increased AVF flow. ${ }^{1}$

We noticed, however, that there are concerns for some biases that could significantly influence the validity of results and consider that the results of study should be interpreted with caution. First, the authors note that 'A left ventricular end-diastolic diameter decrease after operation was predicted by elevated baseline.' Without validation, such a conclusion is an optimistic interpretation. We request that the authors check the validity of the finding with bootstrap resampling.

The authors dichotomized the patients into normal CI and high CI groups. By dichotomizing a group with a continuous baseline, the result can be influenced by regression to mean. We suggest that the authors use the CI index at baseline as continuous and evaluate its discrimination power by using the area under the curve. Additionally, the CI index fluctuates within individuals over time, and overlooking this within-individual variability can lead to regression dilution bias in the results.

Moreover, it seems that there is some degree of over-parameterization in the results of the study. As a general rule, we need at least 10 observations for each variable in regression models. ${ }^{2}$

\section{CONFLICT OF INTEREST}

The authors declare no conflict of interest.

\section{ACKNOWLEDGEMENTS}

The authors would like to thank the statistics consultants of the Research Development Center of Sina Hospital for their technical assistance. This work was not supported by any organization.

Erfan Ayubi ${ }^{1,2}$, Salman Khazaei ${ }^{3}$, Amin Doosti-Irani ${ }^{2}$ and Saeid Safiri ${ }^{4,5}$
${ }^{1}$ Department of Epidemiology, School of Public Health, Shahid Beheshti University of Medical Sciences, Tehran, Iran;

${ }^{2}$ Department of Epidemiology \& Biostatistics, School of Public Health, Tehran University of Medical Sciences, Tehran, Iran;

${ }^{3}$ Department of Epidemiology, School of Public Health, Hamadan University of Medical Sciences, Hamadan, Iran; ${ }^{4}$ Managerial Epidemiology Research Center, Department of Public Health, School of Nursing and Midwifery, Maragheh University of Medical Sciences, Maragheh, Iran and ${ }^{5}$ Road Traffic Injury Research Center, Department of Statistics \& Epidemiology, Tabriz University of Medical Sciences, Tabriz, Iran E-mail: saeidsafiri@gmail.com

1 Wohlfahrt P, Rokosny S, Melenovsky V, Borlaug BA, Pecenkova V, Balaz P. Cardiac remodeling after reduction of high-flow arteriovenous fistulas in end-stage renal disease. Hypertens Res 2016; 39: 654-659.

2 Munro BH. Statistical methods for health care research. Lippincott Williams \& Wilkins: Philadelphia, PA, USA, 2005. 